\title{
RADIO OBSERVATIONS OF THE MARANO FIELD: SUB-MILLIJANSKY SOURCE COUNTS AND SPECTRAL INDEX STUDIES
}

\author{
C. GRUPPIONI AND P. PARMA
}

Istituto di Radioastronomia del CNR, via Gobetti 101, I-40129, Bologna, Italy

AND

H.R. DE RUITER AND G. ZAMORANI

Osservatorio Astronomico di Bologna, via Zamboni 33, I-40126, Bologna, Italy

\section{Introduction}

The Marano Field (centered at $\alpha(2000)=03^{h} 15^{m} 09^{s}, \delta(2000)=-55^{\circ} 13^{\prime}$ $57^{\prime \prime}$ ) is a deep ROSAT field (flux limit $\sim 4 \times 10^{-15} \mathrm{erg}^{-2} \mathrm{~cm}^{-1}$ ), which has been entirely covered by ESO $3.6 \mathrm{~m}$ plates and in the inner part by deep CCD exposures. In order to follow up these data in other wavelength regions, deep radio observations of this field have been carried out with the Australia Telescope Compact Array (ATCA) at 1.370 and $2.378 \mathrm{GHz}$. The minimum reached rms noise value is $\sim 42 \mu \mathrm{Jy}$ at both frequencies. 80 and 45 sources form complete samples above $5.5 \sigma_{\text {local }}$ level at 1.370 and $2.378 \mathrm{GHz}$ respectively, in a square area of $\sim 0.34 \mathrm{sq}$. deg. Almost all of the sources detected at $2.378 \mathrm{GHz}$ have been detected also at $1.370 \mathrm{GHz}$.

\section{The Source Counts}

The normalized source counts at $1.370 \mathrm{GHz}$ show a flattening below a few $\mathrm{mJy}$, equivalent to a more rapid increase in the number of faint sources. This change in slope is visible in all survey fields reaching sub-mJy fluxes at $1.4 \mathrm{GHz}$ (e.g. Condon \& Mitchell 1984; Windhorst et al. 1985). Our normalized differential counts at this frequency are in excellent agreement, both in shape and normalization, with the existing data. 
Our $2.378 \mathrm{GHz}$ counts are the deepest at this or similar frequencies (e.g. $2.7 \mathrm{GHz}$ ). Only predictions based on fluctuation analysis exist at $2.7 \mathrm{GHz}$ down to $\sim 1 \mathrm{mJy}$ (Wall \& Cooke 1975). Our counts are in good agreement with these predictions and with the differential $2.7 \mathrm{GHz}$ counts at higher fluxes (Wall \& Peacork 1985).

\section{Spectral Index Studies}

To study the spectral index distribution, sources which are present in one of the two complete samples but not in the other one have been searched for detection down to the $3 \sigma_{\text {local }}$ level. If no detection was found at this limit, an upper or a lower limit on $\alpha\left(F_{\nu} \sim \nu^{-\alpha}\right)$ was established assuming $S_{1.370}$ or $\mathrm{S}_{2.378}<3 \sigma_{\text {local }}$. The median spectral index for the sources detected at both frequencies is $\alpha_{\text {med }} \simeq 0.59$, but, since most of the limits on $\alpha$ are lower limits, the true $\alpha_{\text {med }}$ is likely to be larger than this value. Above $S_{1.370} \simeq 0.7 \mathrm{mJy}$, where all the sources have been detected at both frequencies, the median spectral index is $0.65 \pm 0.09$, in good agreement with the results discussed by Windhorst et al. (1993). From the analysis of the spectral index as a function of the $1.370 \mathrm{GHz}$ flux density we find that a significant number of inverted spectrum sources $(\alpha<0)$; appears at fluxes below $\sim 2 \mathrm{mJy}$. Spectroscopic identifications of the optical counterparts of these objects are in progress, in order to understand their nature and to investigate the recent results of Windhorst et al. (1995) and Hammer et al. (1995). In particular, the last ones found that $\sim 50 \%$ of the $\mu \mathrm{Jy}$ population with optical counterpart do have inverted radio spectra. About half of them have been identified with low $\mathrm{z}$, low luminosity blue emission line objects, while the remaining ones are red ellipticals at $z>0.75$.

\section{References}

Condon J.J. and Mitchell K.J. (1984), AJ, Vol. no. 89, p. 610.

Hammer F., Crampton D., Lilly S.J., Le Fèvre O. and Kenet T. (1995), MNRAS, Vol. no. 276, p.1085.

Wall I.V. and Cooke D.J. (1975), MNRAS, Vol. no. 171, p. 9.

Wall J.V. and Peacock J.A. (1985), MNRAS, Vol. no. 216, p. 173.

Windhorst R.A., Miley G.K., Owen F.N., Kron R.G. and Koo D.C. (1985), Ap.J., Vol. no. $289, \bar{p} .494$.

Windhorst R.A., Fomalont E.B., Partridge R.B. and Lowenthal J.D. (1993), Ap J., Vol. no. 405 , p. 498.

Windhorst R.A., Fomalont E.B., Kellermann K.I., Partridge R.B., Richards E., Franklin B.E., Pascarelle S.M. and Griffiths R.E. (1995), Nature, Vol. no. 375, p. 471. 